individuelle spécifique à chaque projet issu de ces données et/ou échantillons est difficilement compatible avec les projets reposant sur une réutilisation secondaire renouvelée et réitérée de données ou d'échantillons biologiques faiblement identifiants, collectés à cette fin. C'est pourquoi, il est indispensable de concevoir des modalités d'exercice des droits souples et dynamiques, susceptibles de permettre aux infrastructures de répondre aux enjeux d'une meilleure compréhension des mécanismes pathologiques, tout en garantissant une meilleure maîtrise par les personnes des données et des échantillons biologiques qui les concernent. À cet égard, il faut saluer l'évolution de la doctrine de la CNIL sur ce point, illustrée par le projet de méthodologie de référence MR004 soumis à la concertation des acteurs représentatifs de la recherche. Celle-ci admet que l'information puisse être considérée comme valablement délivrée dès lors que les personnes avaient été informées de la réutilisation possible de leurs données et/ou échantillons biologiques lors de la collecte initiale et que l'information initiale renvoie à un dispositif spécifique d'information auquel les personnes pourront se reporter avant la mise en œuvre d'un nouveau traitement (par exemple un site internet). Ce type d'approche apparaît de nature à favoriser l'utilisation des données en recherche tout en préservant l'autonomie des patients qui deviennent de véritables parties prenantes au projet. Il est également souhaitable que les patients ou leurs représentants intègrent les structures de gouvernance des infrastructures et participent à l'élaboration du projet, des principes directeurs et des procédures d'accès mises en place. $\diamond$

Research in health and personal data: the new situation LIENS D'INTÉRÊT

L'auteur déclare n'avoir aucun lien d'intérêt concernant les données publiées dans cet article.

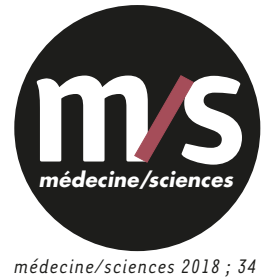
(hors série $n^{\circ} 1$ ) : $28-29$

\section{L'implication du malade expert dans toutes les étapes du développement des thérapies : état des fieux}

Karen Aïach
> Je suis la mère d'une petite fille, atteinte d'une maladie de surcharge lysosomale, le syndrome de Sanfilippo de type A, maladie neurologique et mortelle au début de l'adolescence du patient. J'ai aussi goûté à la vie associative au sein d'Eurordis et au sein d'une association que j'ai créée autour de la maladie de Sanfilippo. J'ai eu une expérience réglementaire au sein du Comité pédiatrique de l'Agence européenne du médicament et d'un comité de protection des personnes. J'ai décidé il y a quelques années de créer une société de biotechnologie pour porter le projet thérapeutique que j'avais mis en œuvre avec d'autres et essayer de mettre au point un traitement pour la maladie de Sanfilippo.

Les réglementations essentielles et fondatrices qui nous permettent d'être ici aujourd'hui ont été en grande partie initiées par des patients, leurs familles et ceux qui les entourent, comme les chercheurs et les médecins. Ces acteurs ont agi dans bien des champs différents, à commencer par la sensibilisation à la maladie rare et par l'éducation. La loi Hôpital, patient, santé et territoire de 2009 fait d'ailleurs essentiellement référence à cette dimension éducative. Le

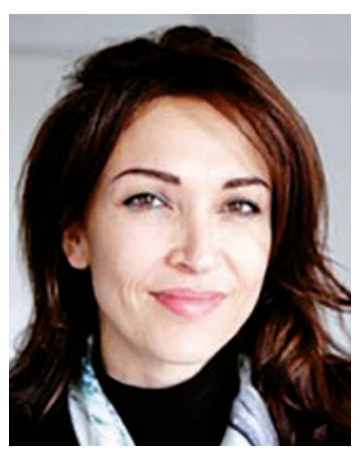

Fondatrice et Directrice Générale, Lysogene, 18 , rue Jacques Dulud, 92200 Neuilly-sur-Seine, France.

karen.aiach@lysogene.fr

patient se retrouve au cœur de l'innovation thérapeutique, non seulement comme objet d'étude, mais aussi comme acteur de l'éducation thérapeutique.

Un vaste écosystème s'est développé autour des patients et des familles, vivace et de grande qualité. Diplômée de I'ESSEC, j'ai commencé ma carrière dans une société américaine et ait travaillé dans le consulting. En arrivant dans l'univers des maladies rares, j'ai été frappée par la qualité des profils et des processus collaboratifs mis en œuvre par les différents acteurs. 
La recherche fondamentale est entre les mains de chercheurs académiques. Ils ne travaillent pas de manière isolée, mais sont souvent en lien avec les associations de patients, notamment dans le champ des maladies rares, au premier chef parce que certains de ces travaux sont financés par les associations de patients. Au-delà, les associations tissent des liens entre patients et chercheurs, mais aussi entre chercheurs et cliniciens. Plus tard dans le développement, au stade dit discovery et préclinique, les patients doivent être impliqués, pour s'assurer que les objectifs poursuivis ont un sens. En effet, on fait des médicaments pour traiter des patients, c'est-à-dire améliorer les conditions de vie de manière significative pour le patient et sa famille. Il est donc impératif de prendre la réalité de leur vie en compte lors du design des études et des essais.

\section{Les enjeux de l'évaluation pour le malade}

Concrètement, les paramètres d'évaluation doivent être significatifs pour le patient. Que le succès de certains essais cliniques repose sur des biomarqueurs est donc source d'interrogation : encore faut-il que ce biomarqueur ait une signification clinique. Lysogene intervient dans une maladie particulièrement compliquée, d'origine neurologique. L'important pour nous n'est pas de déterminer l'évolution d'un biomarqueur donné, mais apporter un bénéfice cognitif à nos patients, ce qui implique de travailler en étroite collaboration avec leurs représentants.

Les patients, leurs familles et les associations ont également pour champ d'action dans le domaine thérapeutique les interactions avec les agences réglementaires. Dans le cadre d'un protocole EUnetHTA, le promoteur que nous sommes, cherche à comprendre ce que les organismes d'évaluation des technologies de la santé attendront de nous lorsque le produit pourra prétendre à une mise sur le marché et une approbation. Nous avons donc rencontré des représentants de patients au travers des hautes autorités de santé. Ces échanges sont fondamentaux et représentent un progrès majeur.

Enfin, il est important que les patients soient actifs dans la phase de pharmacovigilance, une fois le produit sur le marché. Un médicament cible une maladie et des malades, mais il est difficile de dire qu'il sera efficace et sûr pour 100 \% des patients atteints d'une même maladie, rare ou fréquente. Sans entrer dans des polémiques ou des class actions, il est important que se développe et se structure la prise en compte de la voix des patients après la mise sur le marché des produits. On peut ainsi améliorer la prise en charge des patients bénéficiant de ce médicament, exclure du traitement ceux qui n'en bénéficient pas ou l'aménager. Cette proximité permanente des acteurs est donc très importante.

\section{Le travail d'une biotech avec les associations de malades}

Dès les premières heures du programme Sanfilippo, nous avons eu le soutien d'associations de patients. Nous menons actuellement une étude d'histoire naturelle dans plusieurs pays d'Europe et d'Amérique du Sud. Cet essai clinique, mené dans les règles de l'art, est international et multi-centré. II est soumis à un contexte réglementaire très contraignant, car il servira de comparateur à l'essai d'enregistrement de notre premier produit. Nous travaillons avec de jeunes enfants, vulnérables, hyperactifs, qui ne parlent pas, avec lesquels la communication est pratiquement impossible. Nous travaillons avec les familles pour gérer au mieux l'évaluation de ces patients, qu'il est extrêmement difficile de faire voyager. La logistique est donc très lourde. La solution que nous avons trouvée consiste à nouer un partenariat avec des associations de patients pour assurer toute la logistique du départ de l'enfant à l'hôpital jusqu'au retour à la maison.

Enfin, il est important de travailler avec les associations de patients pour identifier les données pertinentes, c'est-à-dire réellement prédictibles ou clairement associées à un bénéfice attendu par le patient. Nous avons d'ailleurs créé un Patient Advisory Board au sein de la société, parallèlement au Clinical Advisory Board. $\diamond$ Place of the expert patient in the R\&D process in the field of rare diseases

\section{LIENS D'INTÉRÊT}

L'auteur déclare n'avoir aucun lien d'intérêt concernant les données publiées dans cet article. 